\title{
EXCEPTIONAL INTEGRALS OF A NOT COMPLETELY INTEGRABLE TOTAL DIFFERENTIAL EQUATION
}

\author{
by H. T. H. PIAGGIO \\ (Received 1st December, 1952)
}

1. There are exceptional integrals of the total differential equation

$$
P(x, y, z) d x+Q(x, y, z) d y+R(x, y, z) d z=0,
$$

in the case when it is not completely integrable, and so when the invariant

$$
I(x, y, z) \equiv P\left(Q_{z}-R_{y}\right)+Q\left(R_{x}-P_{z}\right)+R\left(P_{y}-Q_{x}\right)
$$

is not identically zero, which do not seem to be mentioned by any standard authorities such as Cartan, Goursat, de la Vallée Poussin, and Schouten and Kulk. These are integrals of (1) which do not reduce $I$ to zero. They arise only when the first partial derivates of $P, Q, R$ are not all continuous. A simple example is $z=0$ as an integral of

$$
z^{m} d x+z^{n} d y+d z=0, \quad(m>0, n>0) .
$$

The invariant $I=(n-m) z^{m+n-1}$, which, if $m \neq n$, is a non-zero constant if $m+n=1$, and becomes infinite when $z=0$ if $m+n<1$.

2. It is well known that in general the solution of (1) consists of an infinite number of pairs of equations. Cartan (1) and Goursat (2) give a method of reduction of (1) to a canonical form, and show that this method fails when there are integrals which reduce $I$,to zero. Such integrals they call singular, whether they are of two dimensions (given by a single equation) or of one dimension (given by a pair of equations). Goursat expressly states that he disregards differential equations with non-holomorphic coefficients; though he gives one example, which is completely integrable. De la Vallée Poussin (3) defines a singular integral in a way applicable only to a completely integrable equation. He says that a not completely integrable equation may perhaps be satisfied by $z=\phi(x, y)$, not containing an arbitrary constant, but if so this relation must reduce $I$ to zero. However, on referring back to an earlier paragraph it appears that the discussion is restricted to differential equations whose coefficients have continuous first partial derivatives. Schouten and Kulk (4) do not seem to mention singular integrals at all.

3. We now state the conditions that (1) may be satisfied by a single equation (which we should have called a singular integral if Cartan and Goursat had not used this term in another sense) and investigate why the usual conclusion that it reduces $I$ to zero may be false. The necessary and sufficient conditions that $z=f(x, y)$ should satisfy (1) are

$$
P+R f_{x}=0, \quad Q+R f_{y}=0,
$$

either identically, ${ }^{*}$ or in consequence of $z=f(x, y)$. It is usually impossible for both of equations (2) to be satisfied simultaneously. If they are so satisfied for a function which satisfies the usual relation $f_{x y}=f_{y x}$, we obtain the necessary condition

$$
\frac{\partial}{\partial y}\left(-\frac{p}{r}\right)=\frac{\partial}{\partial x}\left(-\frac{q}{r}\right)
$$

* A sufficient, though not necessary, condition that (1) should be completely integrable is that both of equations (2) should be satisfied identically, for then (1) reduces to $R d\{z-f(x, y)\}=0$. 
where $p, q, r$ denote the results of replacing $z$ by $f(x, y)$ in $P, Q, R$ respectively. Now $p_{\nu}$ can $u s u a l l y$ be obtained by replacing $z$ by $f(x, y)$ in $P_{y}+z_{y} P_{z}$, or, in consequence of (2), in $P_{y}-\frac{Q P_{z}}{R}$.

This is the critical step. If it is valid, together with similar arguments for $q_{x}, r_{x}$ and $r_{y}$, we reach the usual conclusion that $I$ must vanish when $z=f(x, y)$. But in our example $p_{y}=0$, while

$$
P_{v}-\frac{Q P_{z}}{R}=-z^{n} m z^{m-1}
$$

which does not vanish when $z=0$ if $m+n-1 \leqslant 1$. The reasoning fails at the critical step in consequence of the discontinuity of $P_{z}$ when $z=0$, as $m<1$ if $m+n \leqslant 1$ and $n>0$.

4. Hence we have the following theorem :

The necessary condition that $P d x+Q d y+R d z=0$ should be satisfied by a single equation $z=f(x, y)$ is that at least one of the conditions $I=0$, or the discontinuity of one or more first partial derivates of the coefficients $P, Q, R$ should arise when $z=f(x, y)$. Having obtained the function $f(x, y)$ from any of these alternative conditions, we test whether it is an integral or not by the sufficient conditions that it should make both $P+R f_{x}$ and $Q+R f_{y}$ vanish.

If the integral suggested by the necessary conditicn does not contain $z$, we may use the forms $x=g(y, z)$ or $y=h(z, x)$, and make the corresponding modifications in the sufficient conditions.

Additional Examples. The example with which we started can be generalized by introducing other functions, or by transforming the differential equation so that the exceptional integral becomes $z=f(x, y)$.

If $a, b, c$ are non-zero constants, $m, n$ unequal positive constants such that $m+n \leqslant \mathrm{I}$, and $\mu ; \nu$ any constants such that $\mu+\nu>0$, then $z=0$ is an exceptional integral of

$$
a \sin ^{m} z d x+b \sin ^{n} z d y+c d z=0,
$$

for which $I=a b(n-m) \sin ^{m+n-1} z \cos z$, and also of

$$
a z^{m}(\log z)^{\mu}+b z^{n}(\log z)^{\nu}+c d z=0,
$$

for which $I=a b z^{m+n-1}\left\{(n-m)(\log z)^{\mu+v}+(\nu-\mu)(\log z)^{\mu+v-1}\right\}$. More complicated examples can be constructed by replacing the constarts $a, b, c$ by suitable functions of $x, y, z$. These functions and their first partial derivatives should be finite and non-zero when $z=0$.

If, in $a z^{m} d x+b z^{n} d y+c d z=0$, we replace $z$ by $z-f(x, y)$, we obtain

$$
\left[a\{z-f(x, y)\}^{m}-c \frac{\partial f}{\partial x}\right] d x+\left[b\{z-f(x, y)\}^{n}-c \frac{\partial f}{\partial \dot{y}}\right] d y+c d z=0
$$

for which $I=a b(n-m)\{z-f(x, y)\}^{m+n-1}$.

Similarly any other differential equation for which $z=0$ is an exceptional integral can be transformed so that the exceptional integral becomes $z=f(x, y)$.

\section{REFERENCES}

(1) Cartan, E., Ann. Éc. Norm. Sup., (3), 16, 279, 280 (1899).

(2) Goursat, E., Leçons sur le problème de Pfaff (Paris, 1922), pp. 187-190.

(3) de la Vallée Poussin, Ch..J., Cours d'Analyse Infinitésimale, t. II. (7th ed., 1946), pp. 303-4.

(4) Schouten, J. A., and Kulk, W. v. D., Pfaff's Problem and its Generalizations (Oxford, 1949).

\section{UNIVERSTTY OF NOTTINGHAM}

